\title{
Desenvolvimento de objetos de aprendizagem para a matemática utilizando o dispositivo de NUI Leap Motion
}

\author{
Mauro Kolberg Lipp, João Batista Mossmann, Marta Rosecler Bez \\ Instituto de Ciências Exatas e Tecnológicas - Universidade Feevale \\ ERS-239, 2755, Novo Hamburgo - RS - Brazil \\ maurolipp@gmail.com; mossmann@gmail.com; martabez@gmail.com
}

\begin{abstract}
This article aims to present the proposal to use the Leap Motion NUI device. Four learning objects were developed in the mathematics area as a concept proof. The results of the conducted evaluation show that the prototypes have great potential to teach different math contents in a fun, motivating and engaging way.
\end{abstract}

Resumo. Este artigo tem como objetivo apresentar a proposta de uso do dispositivo de NUI Leap Motion. Para tanto foram desenvolvidos quatro objetos de aprendizagem na área de matemática como prova de conceito. Os resultados da avaliação realizada apontam que os protótipos desenvolvidos possuem grande potencial para ensinar diferentes conteúdos de matemática de forma divertida, motivadora e engajante.

\section{Introdução}

Nos últimos anos, o interesse em melhorar a interação entre homens e computadores vem crescendo em diversos aspectos. Discute-se que para atingir real eficiência é necessário criar mecanismos mais naturais ao usuário, se aproximando da forma com que os homens interagem entre si e com objetos do mundo real (SEBE et al., 2004). Neste contexto, o termo Natural User Interface (NUI) emergiu como uma área de Interface Homem-Computador (IHC) que estuda formas do homem interagir com o computador através de suas habilidades naturais (como a fala, a visão, a escrita, o tato, os gestos, os movimentos corporais, entre outros) (NUIGROUP, 2011). Segundo Norman (2010), interações por meio de NUIs possuem um grande potencial, mas também trazem novos problemas e desafios.

Para Wigdor e Wixon (2011), o elemento natural de uma NUI se refere à forma com que o usuário interage com o sistema e como ele se sente nesta interação. Devem ser aproveitadas ao máximo as capacidades do usuário, adaptando-se ao contexto de suas tarefas e demandas. Devem ser evitados longos treinamentos, ou seja, o usuário deve se sentir a vontade desde o primeiro contato. Para que o uso do sistema se mantenha natural, as experiências anteriores e o aprendizado adquirido pelo usuário em relação ao sistema devem ser considerados, permitindo que o mesmo possa evoluir até se tornar um especialista (WIDGOR e WIXON, 2011).

A maioria dos usuários emprega o padrão olhar-mover-olhar, onde o usuário observa a interface, realiza uma ação para interagir sobre ela e, por fim, observa as mudanças realizadas, influenciando na escolha de sua próxima ação (SCHÖN, 1992). 
Bowman et al. (2004) revelaram a dificuldade que muitos usuários possuem em entender espaços tridimensionais. Segundo os autores, isto se deve ao fato de que muitas interpretações do mundo real não podem ser representadas em um ambiente virtual, indicando a necessidade de desenvolvimento de novas metáforas que melhorem a interação e ampliem a compreensão espacial dos usuários.

Este artigo apresenta protótipos de objetos de aprendizagem (WILLEY, 2000), (TAROUCO et al., 2014) desenvolvidos para uso no contexto da matemática utilizado NUI. Na seção 2 é apresentado o dispositivo de NUI utilizado. Os protótipos desenvolvidos são descritos na seção 3 e a validação dos mesmos é apresentada na sessão 4. Por fim, na sessão 5 são discutidas as considerações finais deste estudo.

\section{Tecnologia empregada}

O dispositivo de NUI utilizado neste trabalho é denominado Leap Motion (LEAP MOTION, 2014). Este é um sensor que permite a detecção e rastreamento de mãos e dedos. O Leap Motion realiza o mapeamento de distância dos pontos mais próximos (dentro do seu campo de visão), em relação ao seu ponto central, utilizando feixes de luz infravermelha. A Figura 1 apresenta uma imagem do equipamento e a localização dos eixos cartesianos utilizada pelo mesmo.

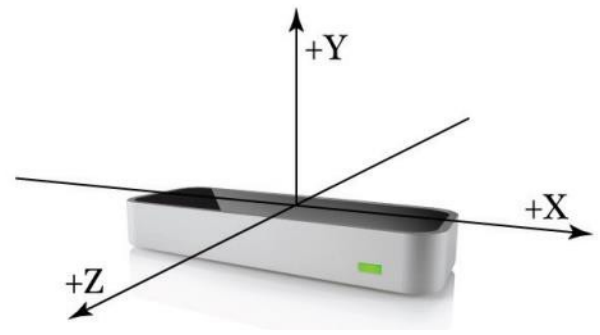

Figura 1 - Localização dos eixos cartesianos em relação ao Leap Motion.

O dispositivo conta com três emissores e dois sensores infravermelhos (Figura 2). O uso de mais emissores e sensores infravermelhos e de um campo de visão reduzido, se comparado ao Kinect (MICROSOFT, 2014), torna o Leap Motion mais preciso. Embora a precisão anunciada pelo fabricante seja de $0,01 \mathrm{~mm}$, testes com versões preliminares do dispositivo indicaram uma precisão média de $0,7 \mathrm{~mm}$ em condições reais. Apesar de representar uma diferença grande entre o anunciado e o obtido efetivamente, a precisão do dispositivo ainda é considerada alta (WEICHERT et al., 2013).

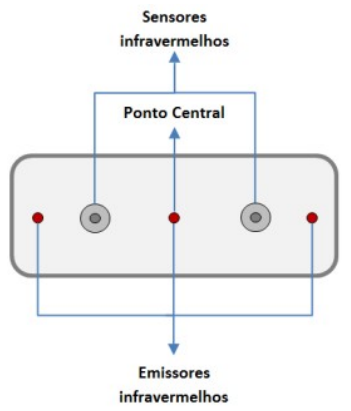

Figura 2 - Tecnologias empregadas no Leap Motion. 


\section{Desenvolvimento de objetos de aprendizagem para prova de conceito do Leap Motion}

A área de aplicação dos objetos de aprendizagem é a matemática. Esta escolha deu-se a partir da percepção da necessidade de pesquisas para a melhoria do ensino nesta área. Prova disso é a baixa classificação da educação brasileira em relação a outros países em indicadores, tal como o realizado pelo Programa Internacional de Avaliação de Estudantes (PISA - Programme for International Student Assessment) (OECD, 2014).

Em 2012, 67,1\% dos estudantes brasileiros ficaram abaixo do nível 2, o que significa que, no máximo, conseguem extrair informações relevantes de uma única fonte, usar algoritmos básicos, fórmulas, procedimentos ou convenções para resolver problemas envolvendo números inteiros. Apenas 1,1\% dos estudantes brasileiros atingiram os níveis 5 e 6 em 2012, contra 12,6\% de estudantes na média geral dos países participantes. Os níveis 5 e 6 indicam que os estudantes possuem capacidade para desenvolver resoluções com modelos em situações complexas e trabalhar estrategicamente usando habilidades de pensamento e raciocínio amplas e bem desenvolvidas, sendo estes os dois maiores níveis que se pode atingir (OECD, 2012).

Dados históricos de outros indicadores, como os produzidos pelo Sistema de Avaliação da Educação Básica (SAEB) em 2001, 2003 e 2005 na avaliação de matemática dos alunos da $4^{\text {a }}$ série de escolas urbanas, apresentam resultados similares à classificação por níveis do PISA descrita anteriormente, com maior concentração de alunos nos níveis mais baixos da escala (SAEB, 2014).

Após o estudo sobre NUI, foram desenvolvidas quatro formas de interação aplicáveis como objetos de aprendizagem na forma de jogos para auxiliar no processo de ensino e aprendizagem de matemática. Uma estratégia adotada no desenvolvimento dos protótipos foi a customização. Definiu-se que os protótipos deveriam ser altamente customizáveis, podendo ser adaptados de forma a abranger diferentes conteúdos e anos escolares, sem a necessidade de se alterar o código-fonte.

Em todos os protótipos os objetivos do jogo são customizáveis, podendo ser ajustados conforme o grau de conhecimento dos jogadores. O objetivo principal é sempre relacionado ao conteúdo que se pretende ensinar. Além do objetivo principal, os protótipos contam com um objetivo secundário: finalizar o nível o mais rápido possível para obter uma medalha de tempo (que pode ser de ouro, prata ou bronze, conforme a velocidade do jogador). A descrição do objetivo principal e o tempo necessário para conquistar cada medalha (que também é customizável) são apresentados ao jogador antes do nível ser iniciado.

Sempre que um jogador informa algum tipo de resposta durante o jogo, lhe é gerado um feedback indicando se sua resposta está correta ou não. Além disso, os protótipos possuem um sistema de pontuação, que mostra o número de acertos e erros do jogador, para que possa acompanhar seu progresso tanto durante a execução do nível, quanto na tela de exibição do resultado final (que é apresentada ao final do nível).

O uso de feedback contínuo também é utilizado para acompanhar o rastreamento das mãos e dedos do jogador. Em todos os protótipos o jogador pode visualizar a posição de suas mãos e dedos detectados pelo dispositivo em tempo real, podendo assim, corrigir movimentos indesejáveis. 
Os protótipos de objetos de aprendizagem desenvolvidos são apresentados na sequência.

\subsection{Maior e Menor}

Neste objeto de aprendizagem dois valores são apresentados na tela ao usuário, um no lado esquerdo e outro no lado direito. O jogador deve usar sua mão para imitar a forma dos símbolos matemáticos de maior e menor para comparar os valores. Se a opção desejada for o símbolo de maior, o jogador deve abrir seu polegar e indicador da mão direita, com o polegar apontando para a esquerda. Para o símbolo de menor, a mesma posição deve ser feita usando a mão esquerda, com o polegar apontando para a direita. Para confirmar a escolha de sua opção, o jogador deve manter a mão na posição por três segundos. A Figura 3 apresenta a tela de interação e um jogador utilizando o objeto de aprendizagem.

Após a confirmação da opção (3 segundo fixos), o sistema indica se o usuário realizou a operação de forma correta e novos valores são apresentados para serem comparados. Quando não houver mais valores a serem exibidos, o nível termina exibindo o tempo total utilizado para finalizá-lo, a medalha de tempo conquistada, o número de comparações corretas e o número de comparações incorretas.

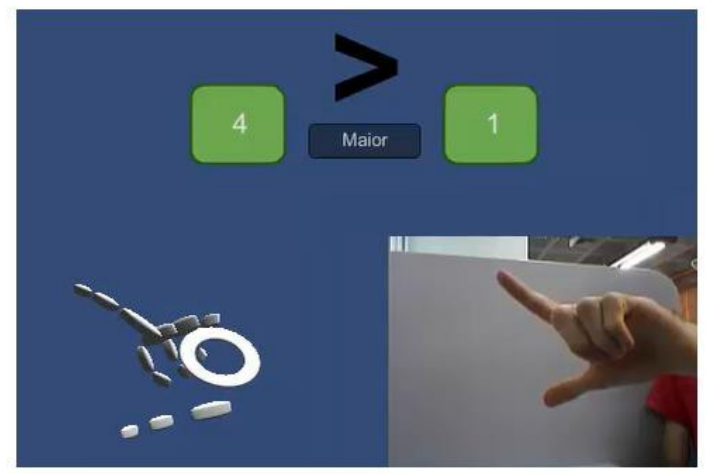

(A)

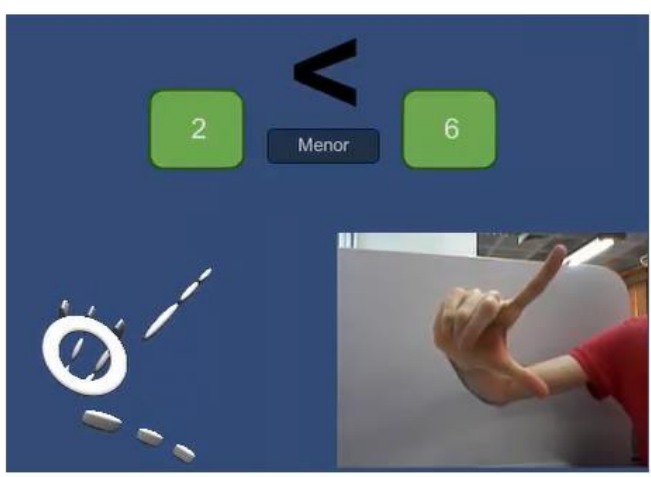

(B)

Figura 3 - Posição da mão para a opção maior (A) e para a opção menor (B).

\subsection{Ordenação de valores}

O objetivo deste objeto de aprendizagem é que o usuário coloque em ordem os elementos apresentados na tela. Para isto ele deve pegar, arrastar e soltar os elementos com a sua mão. A ação de pegar é realizada quando o jogador posiciona sua mão aberta sobre o elemento que deseja pegar e, em seguida, fecha a mão completamente. Enquanto o jogador permanecer com a mão fechada, o elemento será arrastado pela tela seguindo os movimentos de sua mão. Ao abrir a mão, o elemento é solto. A Figura 4 demonstra como estas ações são realizadas.

Quando um elemento é solto em uma posição de troca (posicionado entre dois outros elementos ou ao lado do primeiro ou do último elemento) a aplicação reorganiza a posição de todos os elementos, colocando o elemento solto na posição desejada, e incrementa o número de movimentos realizados. Se o elemento for solto fora de uma 
posição de troca, ele será movido para sua posição anterior (posição que ocupava antes de ser pego pelo jogador).

Quando todos os elementos estiverem na ordem correta, o nível termina mostrando o tempo total utilizado para finalizá-lo, a medalha de tempo conquistada (se houver) e o número de movimentos realizados.
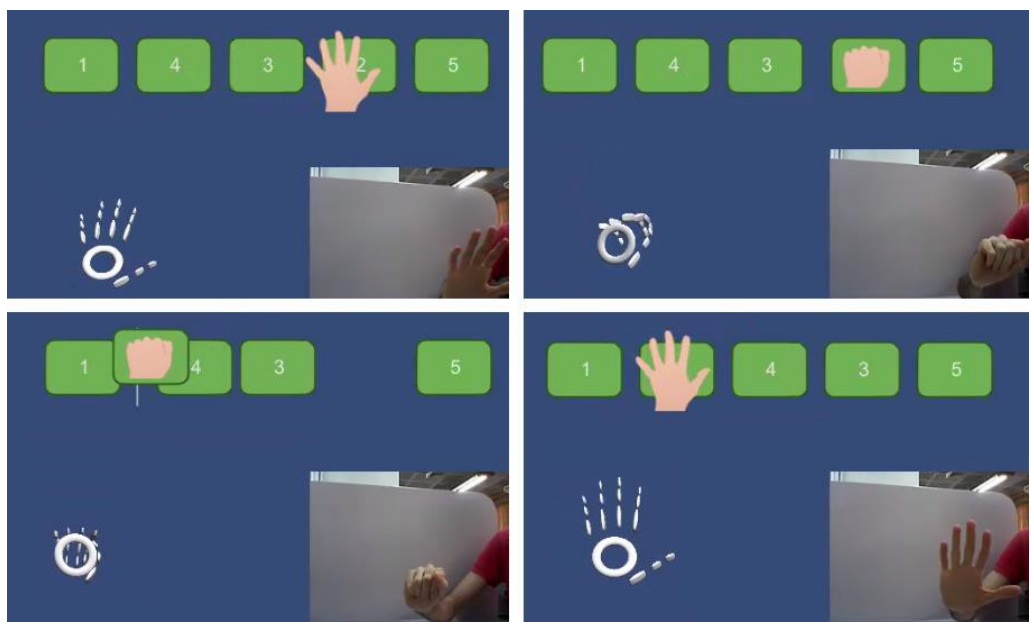

Figura 4 - Movimentos realizados para pegar, arrastar e soltar um elemento.

\subsection{Trabalhando com Medidas}

Neste objeto de aprendizagem o usuário deve tirar as medidas de determinados pontos de uma imagem para resolver problemas matemáticos. Este pode fazer as medições utilizando uma ou as duas mãos. Se apenas uma mão for utilizada, a aplicação exibe a distância entre o dedo polegar e o dedo indicador. Ao usar as duas mãos, a distância exibida é a distância entre os dois dedos indicadores do jogador. A Figura 5 apresenta o objeto de aprendizagem desenvolvido.

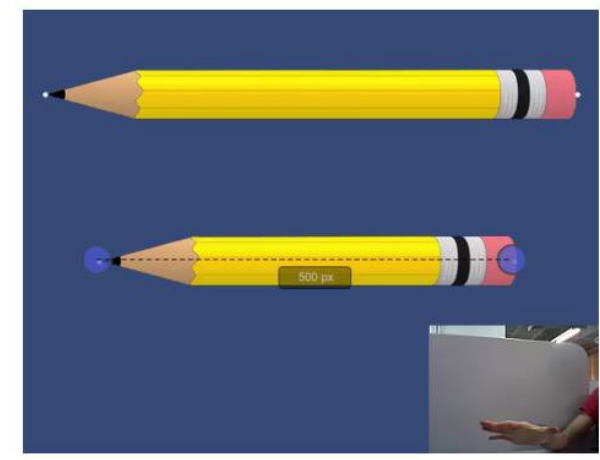

(A)

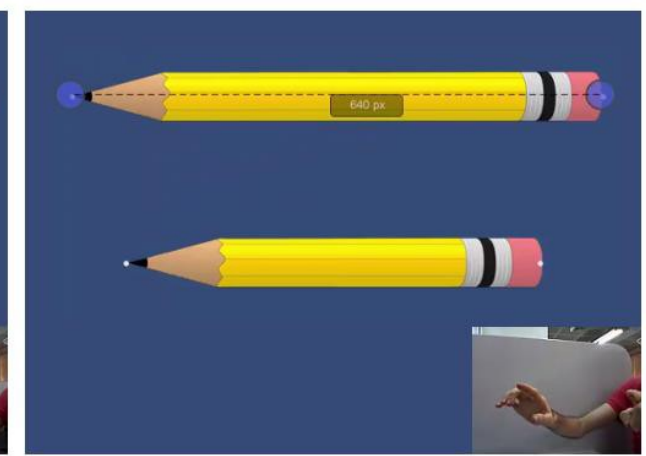

(B)

Figura 5 - Medição com uma mão (A) e com as duas mãos (B).

Se o jogador quiser salvar uma medida, basta que mantenha sua(s) mão(s) parada(s) na posição da medida que pretende salvar durante quatro segundos e a medida ficará visível no canto direito na tela. Assim, poderá consultá-la posteriormente para efetuar os cálculos solicitados no problema. 
Quando o jogador identifica que realizou todas as medidas necessárias para responder o problema proposto, ele deve fazer o gesto de clicar (aproximar e afastar rapidamente o dedo da tela) sobre a barra superior onde o problema está descrito. As alternativas de resposta são apresentadas, e o jogador deve selecionar a que julga que é a alternativa correta (usando o gesto de clicar).

No momento em que uma alternativa é selecionada, o nível é encerrado, mostrando o tempo total utilizado para finalizá-lo e, caso tenha selecionado a alternativa correta, a medalha de tempo conquistada (se houver). Pode-se configurar uma mensagem a ser exibida ao jogador quando a resposta selecionada é a resposta certa (parabenizando o jogador, por exemplo), e uma mensagem quando a resposta está errada (explicando como chegar na solução correta, por exemplo).

\subsection{Classificação}

O objetivo deste objeto de aprendizagem é lançar elementos em seus alvos correspondentes. Pode-se, por exemplo, definir um alvo de números pares e outro de ímpares, fazendo com que o jogador lance os números que lhe são apresentados no alvo correto.

Para lançar um elemento, o jogador deve primeiramente pegá-lo, levando sua mão até ele com os dedos abertos e, em seguida, fazer o movimento de pinçar, aproximando o dedo indicador do polegar até que se encostem. Sem abrir os dedos, o jogador deve puxar o elemento e movimentar sua mão para mirar no alvo desejado. Quanto mais para trás o elemento for puxado, maior será a força aplicada no momento do lançamento. Para lançar o elemento, basta que o jogador abra seus dedos, afastando completamente o dedo indicador do polegar. A Figura 6 apresenta as ações de pegar, puxar e lançar.
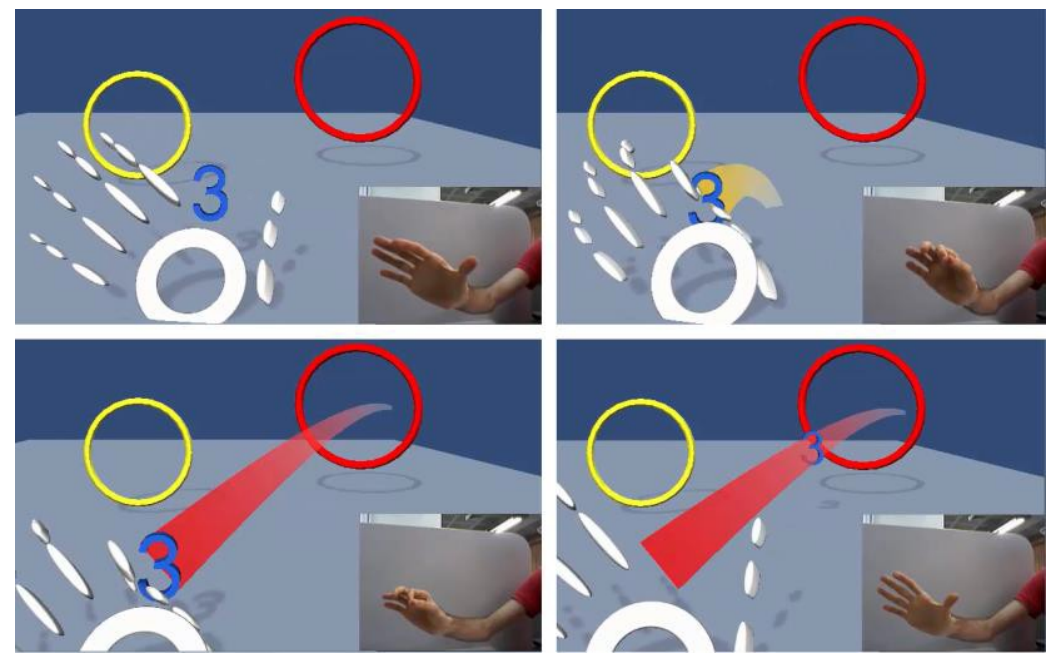

Figura 6 - Ações de pegar, puxar e lançar um elemento.

Quando o elemento lançado atingir um alvo, o sistema irá contabilizar um acerto para o jogador se o alvo atingido for o esperado, ou um erro caso contrário. Se o elemento não atingir nenhum alvo é contabilizada uma jogada "para fora". O nível termina quando não houver mais elementos para se lançar, mostrando o tempo total 
utilizado para finalizá-lo, a medalha de tempo conquistada (se houver), o número de acertos e erros de cada alvo e o número de jogas "para fora”.

Através desta interação pode-se criar jogos que ensinem a classificação de números (pares e ímpares, primos e não primos e naturais, inteiros e reais), de formas geométricas (tipos de triângulos e tipos de polígonos) e até conteúdos de outras áreas do conhecimento como: separação de lixo (em reciclável, não reciclável e orgânico, por exemplo), classificação de animais (vertebrados e invertebrados), classificação de tempos verbais (passado, presente e futuro) ou qualquer outra forma de classificação.

\section{Avaliação}

Após desenvolvidos, os objetos de aprendizagem foram submetidos a um processo de avaliação de usabilidade, utilidade, aceitação e satisfação.

Em razão dos objetos de aprendizagem criados neste trabalho se tratarem de protótipos, optou-se por realizar uma avaliação por especialista. O especialista escolhido possui experiência pedagógica como professor há mais de vinte anos, doutorado em Informática na Educação pela UFRGS e mestrado em Ciência da Computação na PUCRS. Além disso, tem experiência na área de uso/desenvolvimento de tecnologias aplicados nos diferentes níveis de ensino.

Foi criado um questionário para o especialista que utiliza o Technology Acceptance Model (TAM) (VENKATESH; DAVIS, 1996) como forma de avaliar a aceitação (com base nas percepções de facilidade de uso e de utilidade) dos protótipos desenvolvidos e o System Usability Scale (SUS) (BROOKE, 1996) para questões de usabilidade. Além disso, foram adicionadas algumas perguntas descritivas para verificar a satisfação do avaliador em relação ao uso dos protótipos.

Para verificar se o tempo de utilização do Leap Motion poderia influenciar na usabilidade das interações, o questionário foi aplicado em dois momentos distintos: após o primeiro contato com os protótipos e após um período de experimentação de seis dias (com tempo médio de uso de vinte minutos diários). Desta forma, foi possível detectar mudanças de opinião do avaliador após o período de experimentação.

Conforme os resultados apresentados na Figura 7, com exceção do protótipo "Classificação”, a usabilidade dos protótipos após o primeiro contato foi satisfatória, aumentando com certa relevância após o período de experimentação.

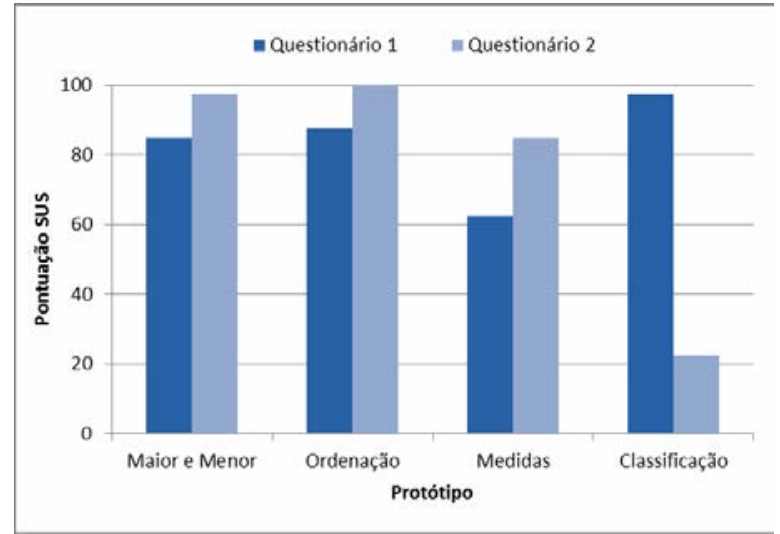

Figura 7 - Pontuação de usabilidade dos protótipos no SUS. 
Em relação à percepção de utilidade, o especialista atribuiu nota máxima em todas as questões para os protótipos avaliados, indicando que possuem potencial para dar auxílio ao ensino de matemática, que podem ser usados para ensinar alunos de diferentes anos escolares e que oferecem motivação e engajamento durante o aprendizado.

Com base nas respostas de percepções de facilidade de uso (usabilidade) e de utilidade presentes no questionário aplicado, foi possível determinar a aceitação do especialista em realação aos protótipos desenvolvidos.

Identificou-se que os protótipos "Maior e Menor” e “Ordenação” foram bem aceitos, sem a necessidade de um período de experimentação. Para o protótipo "Medidas", este resultado pode ser obtido após aplicadas algumas melhorias relacionadas à facilidade de uso, ou após um período de experimentação. Por fim, o protótipo "Classificação" não teve boa aceitação pelo especialista. Isso deve-se a dificuldades relacionadas à perspectiva do ambiente tridimensional do jogo, necessitando de ajustes neste sentido para que tenha maior aceitação.

\section{Considerações finais}

Este artigo apresentou a pesquisa realizada no desenvolvimento de quarto objetos de aprendizagem como prova de conceito que se utilizam de um dispositivo de NUI denominado Leap Motion.

Houve, no desenvolvimento dos protótipos, todo um cuidado em relação ao sentimento do usuário em relação ao sistema, buscando aproveitar ao máximo a capacidade de interação do mesmo através do Leap Motion.

Apesar do foco dado ao desenvolvimento dos protótipos criados neste trabalho ter sido mais direcionado à mecânica das interações, aplicou-se alguns elementos de jogos educacionais (aplicados como objetos de aprendizagem) comuns a todos os protótipos, como: definição de um objetivo, possibilidade de criar níveis com diferentes dificuldades e feedback contínuo para acompanhamento do progresso do aluno.

Cabe ressaltar que, como os jogos são parametrizáveis através de arquivos XML, os mais variados tipos de conteúdos, de diversas áreas, podem ser facilmente incorporados nos objetos de aprendizagem.

Os protótipos foram utilizados por uma semana, no período de 20 minutos por dia por um especialista em Informática na Educação, com o objetivo de avaliar se o uso constante poderia melhorar a interação e analisar o sentimento do mesmo em relação a uma interface não convencional.

Os resultados da avaliação indicam que os protótipos desenvolvidos possuem grande potencial para ensinar diferentes conteúdos de matemática de forma di vertida, motivadora e engajante, embora alguns ajustes sejam necessários. No protótipo "Medidas", identificou-se a necessidade de criar mecanismos que facilitem o entendimento das ações de interação disponíveis. Já no protótipo "Classificação”, devese melhorar a visualização da perspectiva tridimensional do ambiente do jogo. 
Como continuação deste trabalho tem-se a previsão de utilizá-lo com alunos do ensino fundamental, buscando analisar a percepção dos mesmos em relação à interface, bem como os objetos de aprendizagem.

\section{Referencias Bibliográficas}

BOWMAN, D. A.; KRUIJFF, E.; LAVIOLA, J. J. Jr.; POUPYREV, I. (2004) 3D user interfaces: theory and practice. Addison-Wesley.

BROOKE, J. SUS-A quick and dirty usability scale. Usability evaluation in industry, v. 189, p. 194, 1996.

LEAP MOTION. (2014) Leap Motion Documentation. Disponível em: $<$ https://developer.leapmotion.com/documentation/java/index.html>. Acesso em: maio de 2014.

MICROSOFT. Kinect. 2014. Disponível em: <http://www.xbox.com/pt-BR/Home-2>. Acesso em: março de 2014.

NORMAN, D. A. (2010) Natural user interfaces are not natural. interactions, v. 17, n. 3, p. 6-10.

NUIGROUP. Natural User Interface. (2011) Disponível em: <http://wiki.nuigroup.com/Natural_User_Interface>. Acesso em: março de 2014.

OECD. (2014) Programme for International Student Achievement (PISA). Disponível em <http://www.oecd.org/pisa/>. Acesso em: junho de 2014.

OECD. (2012) Programme for International Student Achievement (PISA) - Brazil country notes results from PISA 2012. 2012. Disponível em $<$ http://www.oecd.org/pisa/keyfindings/PISA-2012-results-brazil.pdf $>$. Acesso em: junho de 2014.

SAEB. Sistema de Avaliação da Educação Básica (SAEB). Disponível em: http://portal.inep.gov.br/web/saeb. Acesso em junho de 2014.

SCHÖN, D. A. (1992) Designing as reflective conversation with the materials of a design situation. Knowledge-Based Systems, v. 5, n. 1, p. 3-14.

SEBE, N.; LEW, M. S.; HUANG, T. S. (2004) The state-of-the-art in human-computer interaction. In: Computer Vision in Human-Computer Interaction. Springer Berlin Heidelberg.

TAROUCO, L. M. R.; COSTA, V. M.; ÁVILA, B. G.; BEZ, M. R.; SANTOS, E. F. (2014) Objetos de aprendizagem: teoria e prática. Porto Alegre, Evangraf.

VENKATESH, V.; DAVIS, F. D. A model of the antecedents of perceived ease of use: Development and test. Decision sciences, v. 27, n. 3, p. 451-481, 1996.

WEICHERT, F.; BACHMANN, D.; RUDAK, B.; FISSELER, D. (2013) Analysis of the Accuracy and Robustness of the Leap Motion Controller. Sensors (Basel, Switzerland), v. 13, n. 5, p. 6380.

WIGDOR, D.; WIXON, D. (2011) Brave NUI world: designing natural user interfaces for touch and gesture. Elsevier. 
WILEY, D. (2000) Learning Object Design and Sequencing Theory. Dissertação de Doutorado - Brigham Young University. 2000. Disponível em: $<$ http://opencontent.org/docs/dissertation.pdf>. Acesso em: junho de 2014. 